\title{
CRYSTAL STRUCTURES OF TWO PHOSPHONATE SALTS: MONOCYCLOHEXYLAMMONIUM HYDROGEN PHOSPHONATE AND MONOCYCLOHEXYLAMMONIUM PHENYL PHOSPHONATE
}

\author{
MODOU SARR ${ }^{1}$, MOUHAMADOU BIRAME DIOP ${ }^{*}$, MOUHAMADOU SEMBENE \\ BOYE $^{2}$, AMINATA DIASSE-SARR ${ }^{1}$, PHILIPPE GUIONNEAU ${ }^{3}$ \\ ${ }^{1}$ Cheikh Anta Diop University, Faculty of Science and Technology, Department of \\ Chemistry, Inorganic and Analytical Chemistry Laboratory, Dakar, Senegal \\ ${ }^{2}$ Department of Physics and Chemistry, Faculty of Science and Technology of Education \\ and Training, Cheikh Anta Diop University, Dakar, Senegal \\ ${ }^{3}$ Institue of Solid State Chemistry, CNRS-University of Bordeaux, 87 Avenue Doctor A. \\ Schweitzer 33608 Pessac, France
}

\begin{abstract}
Hydrogen phosphonate anions and monocyclohexylammonium cations interacting through hydrogen bonds conduct to the formation of a salt namely monocyclohexylammonium hydrogen phosphonate. In this structure, hydrogen phosphonate anions are linked by pairs through $\mathrm{O}-\mathrm{H} \cdots \mathrm{O}$ hydrogen bonds leading to anionic dimers. Each dimer is connected to its two neighbours through cations via $\mathrm{N}-\mathrm{H} \cdots \mathrm{O}$ hydrogen bonds leading to infinite chains which are then connected by $\mathrm{N}-\mathrm{H} \cdots \mathrm{O}$ hydrogen bonds giving rise to a layered structure. The phenyl phosphonates form dimers that are connected through an expended hydrogen bonding network involving the cations into a layer.
\end{abstract}

Keywords: crystal structure, monocyclohexylammonium, hydrogen phosphonate, phenyl phosphonate, layered structure

\section{INTRODUCTION}

Interest in phosphonate chemistry is owed to the applications they display $[1,2]$ as well as their properties such as proton conductivity [3,4], optical [5] and catalyst properties [6]. A search of the Cambridge Structural Database (CSD version 5.40) [7] returned 61 different structures containing hydrogen phosphonate anion. 42 of these structures are hydrogen phosphonate ammonium salts and 19 are metallic compounds. Among the 42 ammonium salts, 3 structures contain both hydrogen phosphonate and, fluorophosphonate $\left(\mathrm{FPOH}_{2}{ }^{-}\right)$, phosphonic acid or phosphonate. The CSD shows 20 structures containing hydrogen phenyl phosphonates with an alkaline or an organic counter cation. Five (5) of these hydrogen phenyl phosphonate compounds contain phenyl phosphonic acid molecules. To date, hundreds and hundreds structures of monocyclohexyl ammonium have been deposited in the CSD. The Dakar group focusing in phosphonate characterization has reported several works [810]. Recently, we published the crystalline structures of two triorganotin phosphonate polymers among with the first $\mathrm{PH}_{2}^{-}$bridged triorganotin phosphonate polymer [11]. Thus, continuing to focus in phosphonato organotin(IV), compound $\mathbf{2}$ was first isolated by serendipitous by mixing trimethyltin chloride with monocyclohexyl amine in the presence of phenyl phosphonic acid, $\mathrm{PhPO}(\mathrm{OH})_{2}$. Seeking phosphonate salts soluble in organic solvents usable for further organotin syntheses, we have targeted the interactions between

\footnotetext{
*Corresponding authors, email: mouhamadoubdiop@gmail.com

(C) 2020 Alma Mater Publishing House
} 
monocyclohexyl amine and hydrogen phosphonic acid, $\mathrm{HPO}(\mathrm{OH})_{2}$ leading to the isolation of compound $\mathbf{1}$ (Figure 1). The reaction between monocyclohexyl amine and phenyl phosphonic acid also has been took out yielding crystals of compound $\mathbf{2}$ (Figure 1). We report herein the two structures.

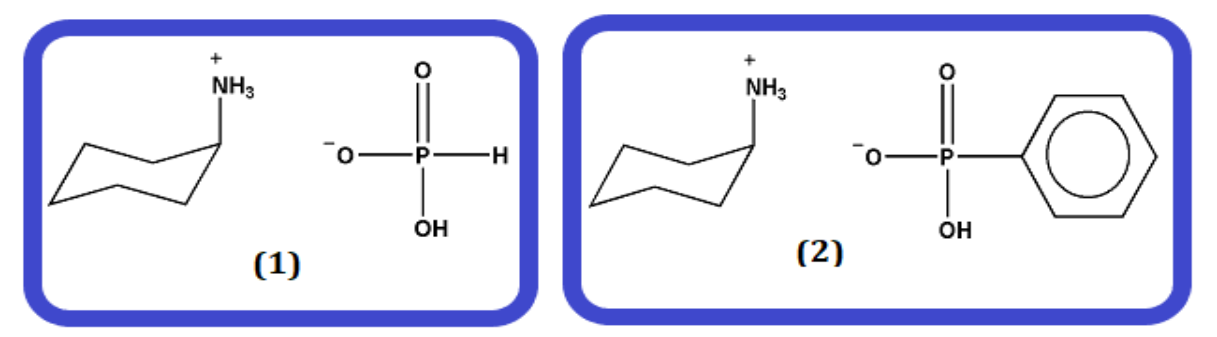

Fig. 1. Molecular representations of monocyclohexylammonium hydrogen phosphonate (1) and monocyclohexylammonium phenyl phosphonate (2) related to this study.

\section{EXPERIMENTAL SETUP}

\section{Synthesis of compounds 1 and 2:}

All chemicals were purchased from Aldrich Company and were used without any further purification. To $20 \mathrm{~mL}$ hydrogen phosphonic acid, $\mathrm{H}_{3} \mathrm{PO}_{3}$ was added slowly $10 \mathrm{~mL}$ equimolar aqueous solution of monocyclohexyl amine, $\mathrm{CyNH}_{2}$. The resulting clear solution was stirred $2 \mathrm{~h}$ at room temperature $(300 \mathrm{~K})$ in the opened atmosphere then submitted to a forced evaporation at $333 \mathrm{~K}$. After some days, colourless block-shaped like crystals of the salt $\mathbf{1}$ suitable for an X-ray crystallographic analysis were obtained. The salt $\mathbf{2}$ was first isolated serendipitously by reacting equimolar amounts of a $5 \mathrm{~mL}$ ethanol solution of monocyclohexyl amine, a $10 \mathrm{~mL}$ ethanol solution of phenylphosphonic acid, and a $15 \mathrm{~mL}$ ethanol solution of trimethyltin chloride. The clear obtained mixture was stirred $2 \mathrm{~h}$ at room temperature $(300 \mathrm{~K})$ in the opened atmosphere. A slow solvent evaporation afforded colourless crystals of salt $\mathbf{2}$ suitable for an X-ray crystallographic study. After its serendipitous synthesis, the salt $\mathbf{2}$ has also been prepared, similarly to salt $\mathbf{1}$, from reaction between phenylphosphonic acid, $\mathrm{PhPO}_{3} \mathrm{H}_{2}$ and monocyclohexyl amine, $\mathrm{CyNH}_{2}$ in a 1:1 molar ratio.

\section{X-ray crystallography of $\left[\left(\mathrm{CyNH}_{3}\right)\left(\mathrm{HPO}_{3} \mathrm{H}\right)\right](1)$ :}

A crystal of approximate dimensions $0.073 \times 0.059 \times 0.011 \mathrm{~mm}$ was used for data collection. The X-ray crystallographic data were collected using a Bruker Kappa ApexII diffractometer, at $T=150 \mathrm{~K}$. Data were measured using $\varphi$ and $\omega$ scans using MoK $\alpha$ radiation $(\lambda=0.71073 \AA)$ using a collection strategy to obtain a hemisphere of unique data determined by Apex2 [12]. Cell parameters were determined and refined using the SAINT program [13]. Data were corrected for absorption by multi-scan absorption correction using SADABS2012/1 [14]. The structure was solved using SHELXT [15] and the structure refined using least-squares minimization SHELXL [16].

Program used for the representation of the molecular and crystal structures: Olex2 [17]. The Crystallographic data and experimental details for structural analyses are summarized in Table 1. Selected bond lengths and angles are listed in Tables 2 and 3, respectively.

CCDC 1978308 (1) contains the supplementary crystallographic data for this paper. These data can be obtained free of charge from the Cambridge Crystallographic Data Centre via www.ccdc.cam.ac.uk/data_request/cif, or by e-mailing data_request@ccdc.cam.ac.uk, or by contacting The Cambridge Crystallographic Data Centre, 12 Union Road, Cambridge CB2 1EZ, UK.

\section{X-ray crystallography of $\left[\left(\mathrm{CyNH}_{3}\right)\left(\mathrm{PhPO}_{3} \mathrm{H}\right)\right](2)$ :}

A crystal of approximate dimensions $0.35 \times 0.25 \times 0.007 \mathrm{~mm}$ was used for data collection. The X-ray crystallographic data were collected using a Bruker Kappa ApexII diffractometer, at $T=150 \mathrm{~K}$. Data were measured using $\varphi$ and $\omega$ scans using $\operatorname{MoK} \alpha$ radiation $(\lambda=0.71073 \AA)$ using a collection strategy to obtain a hemisphere of unique data determined by Apex2 [12]. Cell parameters were determined and refined using the SAINT program [13]. Data were corrected for absorption by multi-scan absorption correction using SADABS2012/1 [14]. The structure was solved using SHELXT [15] and the structure refined using least-squares minimization SHELXL [16]. 
Program used for the representation of the molecular and crystal structures: Olex2 [17]. The Crystallographic data and experimental details for structural analyses are summarized in Table 1. Selected bond lengths and angles are listed in Tables 2 and 3, respectively.

CCDC 1978309 (2) contains the supplementary crystallographic data for this paper. These data can be obtained free of charge from the Cambridge Crystallographic Data Centre via www.ccdc.cam.ac.uk/data_request/cif, or by e-mailing data_request@ccdc.cam.ac.uk, or by contacting The Cambridge Crystallographic Data Centre, 12 Union Road, Cambridge CB2 1EZ, UK.

Table 1. Crystal data and structure refinement for compounds (1) and (2).

\begin{tabular}{|c|c|c|}
\hline Parameters & Compound (1) & Compound (2) \\
\hline \multirow{2}{*}{$\begin{array}{l}\text { Empirical formula } \\
\text { Formula weight }\end{array}$} & $\mathrm{H}_{2} \mathrm{O}_{3} \mathrm{P} \cdot \mathrm{C}_{6} \mathrm{H}_{14} \mathrm{~N}$ & $\mathrm{C}_{6} \mathrm{H}_{6} \mathrm{O}_{3} \mathrm{P} \cdot \mathrm{C}_{6} \mathrm{H}_{14} \mathrm{~N}$ \\
\hline & 181.17 & 257.26 \\
\hline Temperature & $150(2) \mathrm{K}$ & $150(2) \mathrm{K}$ \\
\hline Wavelength & $0.71073 \AA$ & $1.34139 \AA$ \\
\hline Crystal system & Monoclinic & Monoclinic \\
\hline Space group & $P 2_{1} / c$ & $P 2_{1} / c$ \\
\hline \multirow[t]{6}{*}{ Unit cell dimensions } & $a=8.378(11) \AA$ & $\mathrm{a}=13.0297(5) \AA$ \\
\hline & $\alpha=90^{\circ}$ & $\alpha=90^{\circ}$ \\
\hline & $\mathrm{b}=6.623(9) \AA$ & $\mathrm{b}=6.2169(2) \AA$ \\
\hline & $\beta=95.195(17)^{\circ}$ & $\beta=103.043(2)^{\circ}$ \\
\hline & $\mathrm{c}=16.99(2) \AA$ & $\mathrm{c}=17.3831(7) \AA$ \\
\hline & $\gamma=90^{\circ}$ & $\gamma=90^{\circ}$ \\
\hline Volume & $939(2) \AA^{3}$ & $1371.78(9) \AA^{3}$ \\
\hline $\mathrm{Z}$ & 4 & 4 \\
\hline Calculated density & $1.282 \mathrm{~g} \mathrm{~cm}^{-3}$ & $1.246 \mathrm{~g} \mathrm{~cm}^{-3}$ \\
\hline Absorption coefficient & $0.258 \mathrm{~mm}^{-1}$ & $0.198 \mathrm{~mm}^{-1}$ \\
\hline $\mathrm{F}(000)$ & 392 & 552 \\
\hline Crystal size & $0.07 \times 0.06 \times 0.01 \mathrm{~mm}^{3}$ & $0.35 \times 0.25 \times 0.01 \mathrm{~mm}^{3}$ \\
\hline Theta range for data collection & $2.408-27.102^{\circ}$ & $2.405-30.647^{\circ}$ \\
\hline Limiting indices & $\begin{array}{l}-10 \leq h \leq 10,-8 \leq k \leq 8, \\
-21 \leq l \leq 21\end{array}$ & $\begin{array}{l}-18 \leq h \leq 18,-8 \leq k \leq 8 \\
-24 \leq l \leq 24\end{array}$ \\
\hline Reflections collected/unique & $19681 / 2069$ & $44955 / 4217$ \\
\hline $\mathrm{R}_{\text {int }}$ & 0.0671 & 0.0272 \\
\hline Absorption correction & Multi-scan & Multi-scan \\
\hline Max. and min. transmission & 0.7455 and 0.6720 & 0.7461 and 0.7119 \\
\hline Refinement method & Full-matrix least-squares on $F^{2}$ & Full-matrix least-squares on $F^{2}$ \\
\hline Data/restraints/parameters & $2069 / 0 / 153$ & $4217 / 0 / 234$ \\
\hline Goodness-of-fit on $\mathrm{F}^{2}$ & 1.035 & 1.154 \\
\hline Final $\mathrm{R}$ indices $(I>2 \sigma(I))$ & $\mathrm{R}_{1}=0.0377, \mathrm{wR}_{2}=0.0832$ & $\mathrm{R}_{1}=0.0406, \mathrm{wR}_{2}=0.1037$ \\
\hline $\mathrm{R}$ indices (all data) & $\mathrm{R}_{1}=0.0667, \mathrm{wR}_{2}=0.0742$ & $\mathrm{R}_{1}=0.0481, \mathrm{wR}_{2}=0.0976$ \\
\hline Largest diff. peak and hole & 0.275 and $-0.337 \mathrm{e}^{-3}$ & 0.549 and -0.298 e $\AA^{-3}$ \\
\hline
\end{tabular}

Table 2. Selected bond lengths (§) for compounds (1) and (2).

\begin{tabular}{lclc}
\hline & Compound (1) & & Compound (2) \\
\hline Atom-Atom & Bond length & Atom-Atom & Bond length \\
$\mathrm{P} 1-\mathrm{O} 2$ & $1.510(2)$ & $\mathrm{P} 1-\mathrm{O} 3$ & $1.5033(9)$ \\
$\mathrm{P} 1-\mathrm{O} 3$ & $1.505(2)$ & $\mathrm{P} 1-\mathrm{O} 1$ & $1.5063(10)$ \\
$\mathrm{P} 1-\mathrm{O} 1$ & $1.579(2)$ & $\mathrm{P} 1-\mathrm{O} 2$ & $1.5762(9)$ \\
$\mathrm{P} 1-\mathrm{H} 1 \mathrm{P} 1-\mathrm{C} 1$ & $1.8005(13)$ \\
$\mathrm{N} 1-\mathrm{C} 1$ & $1.320(17)$ & $\mathrm{N} 1-\mathrm{C} 7$ & $1.4931(15)$ \\
$\mathrm{C} 1-\mathrm{C} 2$ & $1.505(3)$ & $\mathrm{C} 1-\mathrm{C} 6$ & $1.3898(19)$ \\
$\mathrm{C} 1-\mathrm{C} 6$ & $1.528(3)$ & $\mathrm{C} 1-\mathrm{C} 2$ & $1.3936(19)$ \\
$\mathrm{C} 2-\mathrm{C} 3$ & $1.528(3)$ & $\mathrm{C} 12-\mathrm{C} 7$ & $1.5221(17)$ \\
$\mathrm{C} 6-\mathrm{C} 5$ & $1.534(3)$ & $\mathrm{C} 12-\mathrm{C} 11$ & $1.5252(19)$ \\
$\mathrm{C} 3-\mathrm{C} 4$ & $1.536(3)$ & $\mathrm{C} 7-\mathrm{C} 8$ & $1.5240(17)$ \\
$\mathrm{C} 5-\mathrm{C} 4$ & $1.531(3)$ & $\mathrm{C} 8-\mathrm{C} 9$ & $1.530(2)$ \\
$\mathrm{C} 1-\mathrm{C} 6$ & $1.531(3)$ & $\mathrm{C} 2-\mathrm{C} 5$ & $1.396(2)$ \\
$\mathrm{C} 2-\mathrm{C} 3$ & $1.528(3)$ & $\mathrm{C} 11-\mathrm{C} 3$ & $1.394(2)$ \\
$\mathrm{C} 3-\mathrm{C} 4$ & $\mathrm{C} 9-\mathrm{C} 10$ & $1.524(2)$ \\
$\mathrm{C} 5-\mathrm{C} 4$ & $1.534(3)$ & $\mathrm{C} 3-\mathrm{C} 4$ & $1.526(2)$ \\
$\mathrm{N} 1-\mathrm{C} 1$ & $1.531(3)$ & $\mathrm{C} 4-\mathrm{C} 5$ & $1.378(3)$ \\
$\mathrm{C} 1-\mathrm{C} 2$ & $1.531(3)$ & & $1.380(3)$ \\
$\mathrm{C} 1-\mathrm{C} 6$ & $1.505(3)$ & & \\
\hline
\end{tabular}


Table 3. Selected angles $\left(^{\circ}\right)$ for compounds (1) and (2).

\begin{tabular}{|c|c|c|c|}
\hline \multicolumn{2}{|c|}{ Compound (1) } & \multicolumn{2}{|c|}{ Compound (2) } \\
\hline Atom-atom-atom & Angle value & Atom-atom-atom & Angle value \\
\hline $\mathrm{O} 2-\mathrm{P} 1-\mathrm{O} 1$ & $111.10(8)$ & $\mathrm{O} 3-\mathrm{P} 1-\mathrm{O} 1$ & $116.85(6)$ \\
\hline $\mathrm{O} 2-\mathrm{P} 1-\mathrm{H} 1 \mathrm{D}$ & $105.4(7)$ & $\mathrm{O} 3-\mathrm{P} 1-\mathrm{O} 2$ & $107.09(6)$ \\
\hline $\mathrm{O} 3-\mathrm{P} 1-\mathrm{O} 2$ & $116.65(9)$ & $\mathrm{O} 3-\mathrm{P} 1-\mathrm{C} 1$ & $107.22(6)$ \\
\hline $\mathrm{O} 3-\mathrm{P} 1-\mathrm{O} 1$ & $108.03(10)$ & $\mathrm{O} 1-\mathrm{P} 1-\mathrm{O} 2$ & $110.33(5)$ \\
\hline $\mathrm{O} 3-\mathrm{P} 1-\mathrm{H} 1 \mathrm{D}$ & $109.1(7)$ & $\mathrm{O} 1-\mathrm{P} 1-\mathrm{C} 1$ & $108.40(6)$ \\
\hline $\mathrm{O} 1-\mathrm{P} 1-\mathrm{H} 1 \mathrm{D}$ & $106.0(7)$ & $\mathrm{O} 2-\mathrm{P} 1-\mathrm{C} 1$ & $106.43(6)$ \\
\hline $\mathrm{N} 1-\mathrm{C} 1-\mathrm{C} 2$ & $109.93(16)$ & $\mathrm{C} 6-\mathrm{C} 1-\mathrm{P} 1$ & $121.21(10)$ \\
\hline $\mathrm{N} 1-\mathrm{C} 1-\mathrm{C} 6$ & $110.44(16)$ & $\mathrm{C} 6-\mathrm{C} 1-\mathrm{C} 2$ & $118.75(13)$ \\
\hline $\mathrm{C} 6-\mathrm{C} 1-\mathrm{C} 2$ & $111.52(18)$ & $\mathrm{C} 2-\mathrm{C} 1-\mathrm{P} 1$ & $120.02(11)$ \\
\hline $\mathrm{C} 1-\mathrm{C} 2-\mathrm{C} 3$ & $110.27(17)$ & $\mathrm{C} 7-\mathrm{C} 12-\mathrm{C} 11$ & $110.32(11)$ \\
\hline $\mathrm{C} 1-\mathrm{C} 6-\mathrm{C} 5$ & $110.41(17)$ & $\mathrm{N} 1-\mathrm{C} 7-\mathrm{C} 12$ & $109.63(10)$ \\
\hline $\mathrm{C} 4-\mathrm{C} 3-\mathrm{C} 2$ & $111.26(18)$ & $\mathrm{N} 1-\mathrm{C} 7-\mathrm{C} 8$ & $109.24(10)$ \\
\hline $\mathrm{C} 4-\mathrm{C} 5-\mathrm{C} 6$ & 111.68 (19) & $\mathrm{C} 12-\mathrm{C} 7-\mathrm{C} 8$ & $111.74(10)$ \\
\hline \multirow[t]{9}{*}{$\mathrm{C} 5-\mathrm{C} 4-\mathrm{C} 3$} & $111.6(2)$ & $\mathrm{C} 7-\mathrm{C} 8-\mathrm{C} 9$ & $110.80(12)$ \\
\hline & & $\mathrm{C} 1-\mathrm{C} 6-\mathrm{C} 5$ & $120.50(15)$ \\
\hline & & $\mathrm{C} 1-\mathrm{C} 2-\mathrm{C} 3$ & $120.56(15)$ \\
\hline & & $\mathrm{C} 10-\mathrm{C} 11-\mathrm{C} 12$ & $111.21(13)$ \\
\hline & & $\mathrm{C} 10-\mathrm{C} 9-\mathrm{C} 8$ & $111.20(12)$ \\
\hline & & $\mathrm{C} 4-\mathrm{C} 3-\mathrm{C} 2$ & $120.01(15)$ \\
\hline & & $\mathrm{C} 11-\mathrm{C} 10-\mathrm{C} 9$ & $111.21(13)$ \\
\hline & & $\mathrm{C} 3-\mathrm{C} 4-\mathrm{C} 5$ & $120.16(15)$ \\
\hline & & $\mathrm{C} 4-\mathrm{C} 5-\mathrm{C} 6$ & $120.02(17)$ \\
\hline
\end{tabular}

\section{RESULTS AND DISCUSSION}

Salt 1 crystallizes in the monoclinic space group P2(1)/c. Its asymmetric unit depicted in Figure 2 is comprised of one hydrogen phosphonate anion and one monocyclohexyl ammonium cation. The hydrogen phosphonate anion with $\mathrm{P}-\mathrm{O}$ distances ranging from 1.505 (2) $\AA$ to 1.579 (2) $\AA$ and angles comprised between 105.04 (7) and $116.65(9)^{\circ}$ describes a distorted tetrahedral geometry and are in the range of those previously reported [1820]. The $\mathrm{O}$ atoms of the longer $\mathrm{P}-\mathrm{O}$ bond are both involved in a very strong hydrogen bonding interaction [9, $10,21]$, the significantly longer one being involved in a second strong hydrogen bond. The shortest $\mathrm{P}-\mathrm{O}$ bond is that whose $\mathrm{O}$ atom act as acceptor of two symmetric hydrogen bonds. Bonds and angles within the monocyclohexyl ammonium are otherwise comparable to those recorded in the literature [22-24].

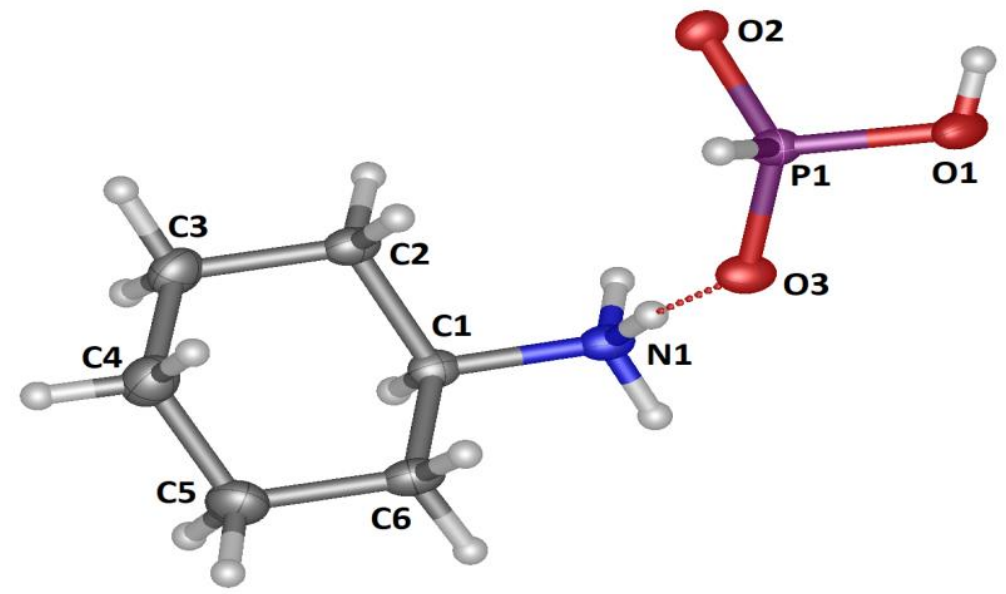

Fig. 2. The molecular structure of compound 1, showing the atom labelling. Displacement ellipsoids are drawn at the $50 \%$ probability level.

Salt 2 crystallizes in the monoclinic space group P2(1)/c. Its asymmetric unit depicted in Figure 3 consists of one phenyl phosphonate anion and one monocyclohexyl ammonium cation. The geometric parameters within the anion with $\mathrm{P}-\mathrm{O}$ lengths varying from 1.5033 (9) $\AA$ to 1.5762 (9) $\AA$ and angles from $106.43(6)^{\circ}$ to $116.85(6)^{\circ}$ are in accordance with those in salt $\mathbf{1}$. The geometric parameters within the cation are otherwise not exceptional compared to those in compound $\mathbf{1}$. 


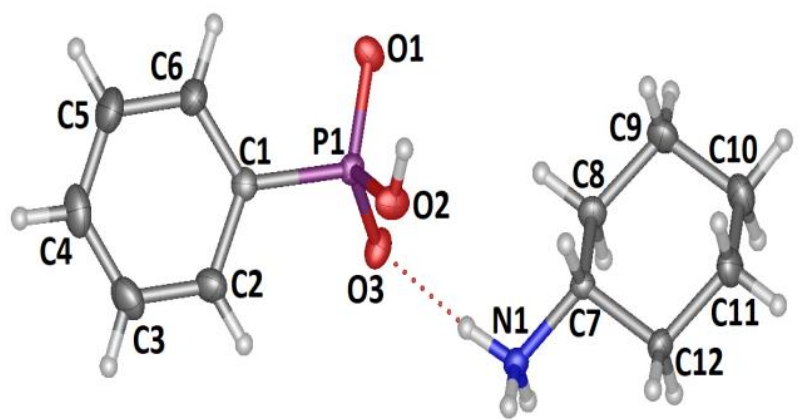

Fig. 3. The molecular structure of compound 2, showing the atom labelling. Displacement ellipsoids are drawn at the $50 \%$ probability level.

In a supramolecular viewing, in the crystal of compounds $\mathbf{1}$ and $\mathbf{2}$, phosphonate anions form dianionic dimers through a very strong $\mathrm{O}-\mathrm{H} \cdots \mathrm{O}$ hydrogen bonding interactions. These dimers are connected to each other through two monocyclohexyl ammonium cations via $\mathrm{N}-\mathrm{H} \cdots \mathrm{O}$ hydrogen bonds growing an hydrogen bonded chain depicted in Figure 4. These chains are then connected, through the remaining nitrogen $\mathrm{H}$ atom of the cation, by $\mathrm{N}-\mathrm{H} \cdots \mathrm{O}$ hydrogen bonds giving rise to a layered structure (Tables 4, 5 and Figure 4). The layer morphologies exhibit two types of self-assemblies describing a 10-membered and a 16-membered hydrogen bonded macrocycles involving both cations and anions, and a 8-membered hydrogen bonded ring involving only anions. The 16-membered and 8-membered hydrogen bonded self-assemblies alternate into the expending chain while the encountered 10-membered hydrogen bonded macrocycle come from linkage of chains that grows the layer. Thus, each cation is surrounded by three different dimers and each dimer is surrounded by six different cations. All the hydrogen bonded macrocycles are both fused (Figure 4).

Table 4. Hydrogen-bond geometry $\left(\AA,^{\circ}\right)$ for compound (1).

\begin{tabular}{lllll}
\hline$D-\mathrm{H} \cdots A$ & $D-\mathrm{H}$ & $\mathrm{H} \cdots A$ & $D \cdots A$ & $D-\mathrm{H} \cdots A$ \\
\hline $\mathrm{N} 1-\mathrm{H} 1 A \cdots \mathrm{O} 2^{\mathrm{i}}$ & 0.91 & 1.89 & $2.799(3)$ & 173 \\
$\mathrm{~N} 1-\mathrm{H} 1 B \cdots \mathrm{O} 3$ & 0.91 & 1.87 & $2.781(4)$ & 175 \\
$\mathrm{~N} 1-\mathrm{H} 1 C \cdots \mathrm{O} 3^{\mathrm{ii}}$ & 0.91 & 1.88 & $2.787(4)$ & 173 \\
$\mathrm{O} 1-\mathrm{H} 1 E \cdots \mathrm{O} 2^{\mathrm{iii}}$ & $0.76(2)$ & $1.88(2)$ & $2.635(3)$ & $173(2)$ \\
\hline
\end{tabular}

Symmetry codes: (i) $-x+2, y-1 / 2,-z+1 / 2$; (ii) $-x+2, y+1 / 2,-z+1 / 2$; (iii) $-x+2,-y+1,-z+1$

Table 5. Hydrogen-bond geometry $\left(\AA{ }^{\circ}\right)$ for compound (2).

\begin{tabular}{lllll}
\hline$D-\mathrm{H} \cdots A$ & $D-\mathrm{H}$ & $\mathrm{H} \cdots A$ & $D \cdots A$ & $D-\mathrm{H} \cdots A$ \\
\hline $\mathrm{N} 1-\mathrm{H} 1 A \cdots \mathrm{O} 3^{\mathrm{i}}$ & $0.884(19)$ & $1.867(19)$ & $2.7497(13)$ & $176.3(18)$ \\
$\mathrm{N} 1-\mathrm{H} 1 B \cdots \mathrm{O} 3$ & $0.94(2)$ & $1.82(2)$ & $2.7511(14)$ & $172.8(18)$ \\
$\mathrm{N} 1-\mathrm{H} 1 C \cdots \mathrm{O} 1^{\mathrm{ii}}$ & $0.90(2)$ & $1.85(2)$ & $2.7470(13)$ & $173.2(18)$ \\
$\mathrm{O} 2-\mathrm{H} 2 \cdots \mathrm{O} 1^{\mathrm{iii}}$ & $0.83(2)$ & $1.76(2)$ & $2.5870(13)$ & $173(2)$ \\
\hline
\end{tabular}

Symmetry codes: (i) $-x+1, y-1 / 2,-z+3 / 2$; (ii) $x, y-1, z$; (iii) $-x+1,-y+1,-z+1$

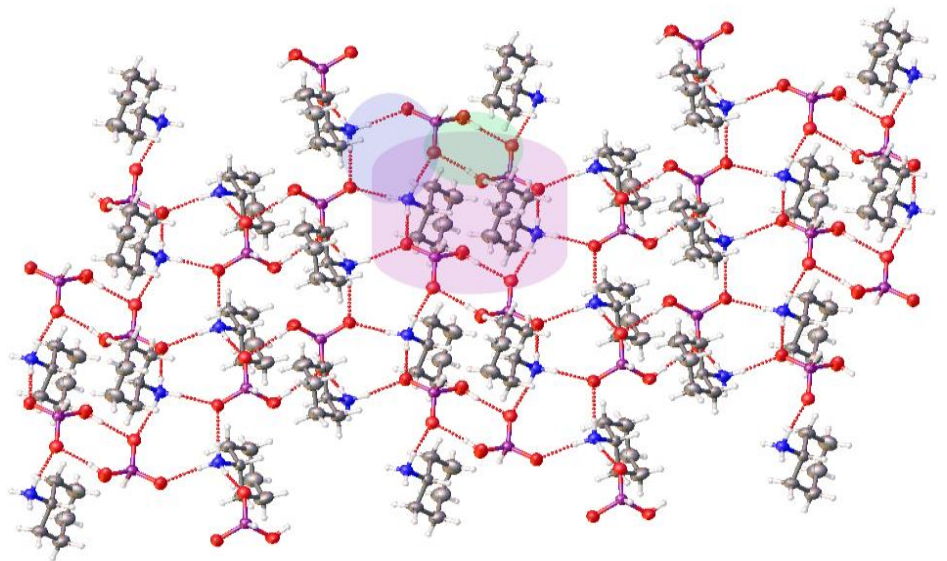

Fig. 4. The molecular structure of salt 1, showing the 16-membered (pink buckle), 10-membered (blue buckle) and 8-membered hydrogen (green buckle) bonded macrocycles within a two-dimensional layer-like arrangement through $\mathrm{N}-\mathrm{H} \cdots \mathrm{O}$ and $\mathrm{O}-\mathrm{H} \cdots \mathrm{O}$ interactions. 
Moreover, in compound 2, the two phenyl groups of the anionic dimers are oriented in such way that they lay in almost coplanar planes so that the former positioned on one side of the 8-membered hydrogen bonded ring plane while the latter occupy the over side. Therefore, all dimers are oriented in an isotactic fashion. Similarly, cyclohexyl groups within compounds $\mathbf{1}$ and $\mathbf{2}$ are also disposed, between dimers, in an up-down sequence that can be described as isotactic (Figure 5).

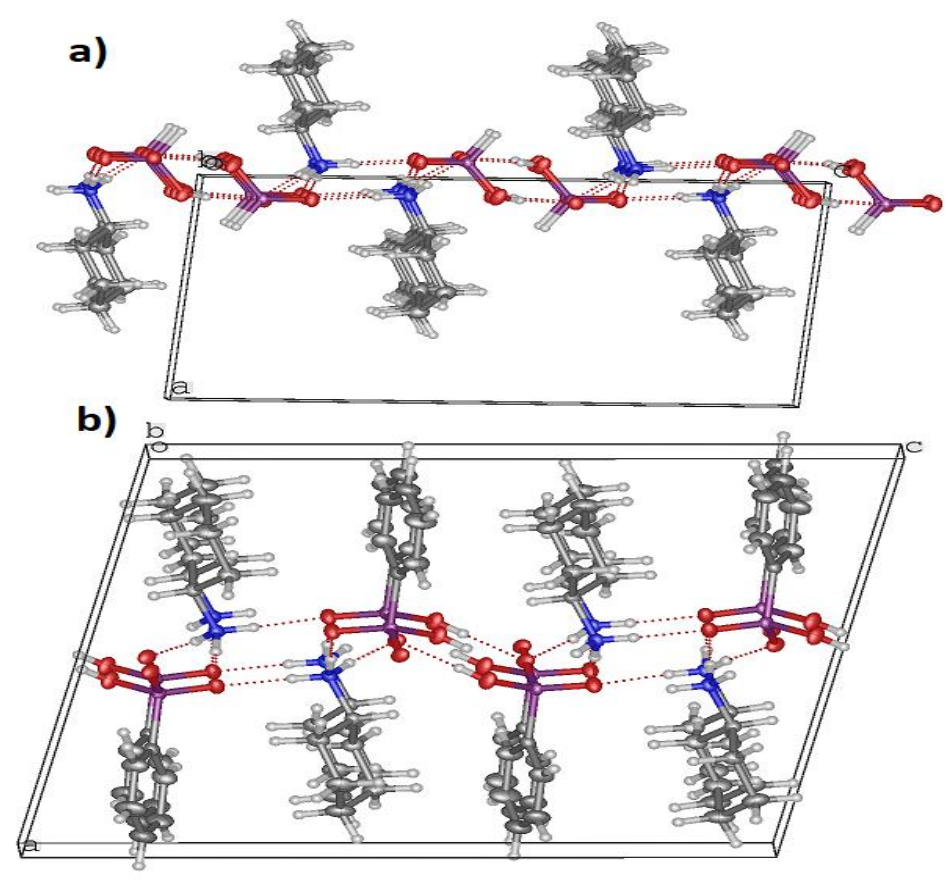

Fig. 5. The crystal packing of salts $\mathbf{1}[\mathrm{a})]$ and $\mathbf{2}[\mathrm{b})]$. Displacement ellipsoids are drawn at the 50\% probability level.

\section{CONCLUSIONS}

The studied phosphonates salts $\mathbf{1}$ and $\mathbf{2}$ both describe a dianionic dimer in which two phosphonate anions are linked through strong H-bonds. In all structures concerning this work, a hydrogen bonded infinite chain grew from hydrogen bonding interactions between dimers and monocycohexyl ammonium. In spite of the formula difference, compounds $\mathbf{1}$ and $\mathbf{2}$ are isostructural. Further attempts investigating the isolation of various metal phosphonate compounds are in progress.

\section{ACKNOWLEDGEMENTS}

The authors acknowledge the Cheikh Anta Diop University of Dakar (Senegal) and the Institute of Solid State Chemistry, Bordeaux (France), for financial support and instrumentation facilities.

\section{REFERENCES}

[1] Abraham, C., Konstantinos, D., Metal phosphonate chemistry: from synthesis to applications, Ed. Royal Society of Chemistry, 2012.

[2] Troev, K.D., Chemistry and application of H-phosphonates, $1^{\text {st }}$ Edition, Ed. Elsevier, 2006, p. 253-284.

[3] Bao, S.-S., Shimizu, G.K.H., Zheng, L.-M., Proton conductive metal phosphonate frameworks, Coordination Chemistry Reviews, vol. 378, 2019, p. 577-594.

[4] Zhang, Y., Jua, J., Li, T., Zhang, Y., Lin, Z., Cai, H., Organically templated metal phosphites: Ionothermal synthesis, crystal structure, and proton conduction, Inorganic Chemistry Communications, vol. 107, 2019, p. 107476. 
[5] Zahid, I.M., Kumar, C.A., Malarkodi, A., Wilson, K.S.J., Kumar, R.M., Studies on the crystal growth and optical properties of novel organic material: O-Phenylenediaminium bis-hydrogen phosphonate, Materials Letters, vol. 209, 2017, p. 167-170.

[6] Wang, B.-L., Zhang, J.-X., Li, N.-K., Liu, G.-G., Shen, Q., Wang, X.-W., Organic hydrogen phosphites and hydrogen phosphates catalyzed Friedel-Crafts amidoalkylation of indoles with aryl aldimines, Tetrahedron Letters, vol. 52, no. 36, 2011, p. 4671-4674.

[7] Groom, C.R., Bruno, I.J., Lightfoot, M.P., Ward, S.C., The cambridge structural database, Acta Crystallographica, vol. B72, no. 2, 2016, p. 171-179.

[8] Diop, C.A.K., Diop, L., Russo, U., $\left(\mathrm{Ph}_{3} \mathrm{Sn}\right)_{2} \mathrm{~A}^{\prime}\left(\mathrm{A}^{\prime}=\mathrm{O}_{4} \mathrm{C}_{2}, \mathrm{O}_{3} \mathrm{Se}, \mathrm{O}_{3} \mathrm{PH}, \mathrm{O}_{3} \mathrm{AsPh}, \mathrm{O}_{3} \mathrm{PCH}_{3}\right.$ and $\left(\mathrm{Ph}_{3} \mathrm{Sn}\right)_{3} \mathrm{O}_{4} \mathrm{P}$ : Synthesis, Mössbauer, IR and NMR studies, Main Group Metal Chemistry, vol. 22, no. 4, 1999, p. 217-220.

[9] Diop, T., Diop, L., Maris, T., Stoeckli-Evans, H., Dicyclohexylammonium hydrogen phenylphosphonate, Acta Crystallographica, vol. E68, no. 5, 2012, p. ol432.

[10] Sarr, M., Boye, M.S., Sarr, A.D., Grosjean, A., Guionneau, P., diisopropylammonium hydrogen phenylphosphonate, Acta Crystallographica, vol. E68, no. 11, 2012, p. o3078.

[11] Diop, M.B., Boye, M.S., Diasse-Sarr, A., Diop, L., Guionneau, P., Maris, T., Triorganotin phosphonates polymeric chains - Synthesis, Infrared, Mössbauer and Single Crystal Characterization: the First organotin(IV) $\mathrm{PH}_{2}^{-}$bridged, International Journal of Advanced Research, vol. 7, no. 8, 2019, p. 266-278.

[12] APEX2 (Version 2013.12), Crystallographic Software, Suite, Bruker AXS Inc., Madison, Wisconsin, USA, 2012.

[13] SAINT (Version 8.34A), Area Detector Integration Software, Bruker AXS Inc., Madison, Wisconsin, USA, 2012.

[14] SADABS (Version 2012/1), Bruker AXS Inc., Madison, Wisconsin, USA, 2012.

[15] Sheldrick, G.M., SHELXT-Integrated space-group and crystal structure determination, Acta Crystallographica, vol. A71, no. 1, 2015, p. 3-8.

[16] Sheldrick, G.M., Crystal structure refinement with SHELXL, Acta Crystallographica, vol. C71, no. 1, 2015, p. 3-8.

[17] Dolomanov, O.V., Bourhis, L.J., Gildea, R.J., Howard, J.A.K., Puschmann, H., OLEX2: a complete structure solution, refinement and analysis program, Journal of Applied Crystallography, vol. 42, no. 2, 2009 , p. 339-341.

[18] Silva, M.R., Paixao, J.A., Beja, A.M., Crystal structure of L-tryptophanium phosphite, $\left(\mathrm{C}_{11} \mathrm{H}_{13} \mathrm{~N}_{2} \mathrm{O}_{2}\right)\left[\mathrm{H}_{2} \mathrm{PO}_{3}\right]$, Zeitschrift fr Kristallographie - New Crystal Structures, vol. 220, 2005, p. 487-488.

[19] Ghazaryan, V.V., Zakharov, B.A., Petrosyan, A.M., Boldyreva, E.V., L-Argininium phosphite - a new candidate for NLO materials, Acta Crystallographica, vol. C71, no. 5, 2015, p. 415-421.

[20] Abu-Salem, Q., Sweidan, K., Mallah, E., Joshi, R., Mubarak, M.S., Steinmann, M., Voelter, W., Hydrogenbonded phosphorous acids. synthesis and structure of imidazolium-containing salts of hydrogenphosphonate and phenylphosphonate, Jordan Journal of Chemistry, vol. 6, no. 2, 2011, p. 113-121.

[21] Beckmann, J., Dakternieks, D., Duthie, A., Crystallographic report: Dimethylammonium phenylphosphonate.2(phenylphosphonic acid, Applied Organometetallic Chemistry, vol. 17, no. 10, 2003 , p. 817-818.

[22] Lemmerer, A., Two-dimensional hydrogen-bonding patterns in a series of salts of terephthalic acid and the cyclic amines $\mathrm{C}_{\mathrm{n}} \mathrm{H}_{2 \mathrm{n}-1} \mathrm{NH}_{2}, \mathrm{n}=3,4,5,6,7,8$, and 12, Crystal Growth and Design, vol. 11, no. 2, 2011, p. 583593.

[23] Grimball, B., Veillon, L., Calhoun, T., Fronczek, F.R., Arceneaux, E., Laine, R.A., Cyclohexylamine inexplicably induces antennae loss in formosan subterranean termites (Coptotermes formosanus Shiraki): cyclohexylamine hydrogen phosphate salts are novel termiticides, Pest Management Science, vol. 73, no. 10, 2017, p. 2039-2047.

[24] Tang, Y.-Z., Yu, Y.-M., Xiong, J.-B., Tan, Y.-H., Wen, H.-R., Unusual high temperature reversible phase transition behaviour, structures and dielectric-ferroelectric properties of two new crown ether clathrates, Journal of the American Chemical Society, vol. 137, no. 41, 2015, p. 13345-13351. 